\title{
Rapid Adsorption of Proinflammatory Cytokines by Graphene Nanoplatelets and Their Composites for Extracorporeal Detoxification
}

\author{
Yishan Zheng, ${ }^{1}$ Nicholas Pescatore, ${ }^{2}$ Yury Gogotsi, ${ }^{2}$ Boris Dyatkin,,3 \\ Ganesh Ingavle, ${ }^{1}$ Vadym Mochalin $\left(\mathbb{D},{ }^{4}\right.$ Tochukwu Ozulumba, \\ Sergey Mikhalovsky, ${ }^{1}$ and Susan Sandeman ${ }^{1}{ }^{1}$ \\ ${ }^{1}$ School of Pharmacy and Biomolecular Sciences, University of Brighton, Brighton BN2 4GJ, UK \\ ${ }^{2}$ Department of Materials Science \& Engineering and A. J. Drexel Nanomaterials Institute, Drexel University, \\ Philadelphia, PA 19104, USA \\ ${ }^{3}$ Chemistry Division, U.S. Naval Research Laboratory, Washington, DC 20375, USA \\ ${ }^{4}$ Department of Chemistry and Department of Materials Science \& Engineering, Missouri University of Science \& Technology, \\ Rolla, MO 65409, USA
}

Correspondence should be addressed to Susan Sandeman; s.sandeman@brighton.ac.uk

Received 19 July 2017; Revised 11 January 2018; Accepted 22 January 2018; Published 21 February 2018

Academic Editor: Renyun Zhang

Copyright (C) 2018 Yishan Zheng et al. This is an open access article distributed under the Creative Commons Attribution License, which permits unrestricted use, distribution, and reproduction in any medium, provided the original work is properly cited.

\begin{abstract}
Sepsis is a complex clinical syndrome that features excessive release of cytokines and other inflammatory mediators that could lead to organ dysfunction. Despite different treatment and management options, sepsis associated high morbidity and mortality rates remain. This has prompted intensive research into alternative therapeutic approaches such as targeted removal of sepsis related molecules using extracorporeal hemoperfusion. In this study, we explore the use of graphene nanoplatelets (GNP) as lowcost alternative hemosorbents for rapid removal of a broad spectrum of proinflammatory cytokine markers. Firstly, the physical characteristics, cytotoxicity, and cytokine marker adsorption profile of GNP were assessed. The results not only confirmed the surface characteristics of GNP and their ability to rapidly remove cytokine markers, but also indicated a low cytotoxicity towards the hepatic cell line HepG2. GNP were then incorporated into a freestanding flexible GNP-poly(tetrafluoroethylene) film with preserved surface characteristics and cytokine adsorption profile for potential use in hemoperfusion applications.
\end{abstract}

\section{Introduction}

Sepsis is a life-threatening condition caused by the body's disregulated host response to infection. Between 1993 and 2003, the number of hospitalizations for severe sepsis doubled in the United States [1]. Sepsis is the primary cause of death for children and infants $[2,3]$, and the number of incidences was estimated at over 19 million cases worldwide annually [4]. Sepsis conditions progress as a function of the cytokine cascade, an exaggerated immune response to the incident infection [5]. Although the exact mechanism of sepsis remains poorly understood, it is believed that high concentrations of proinflammatory cytokines, such as IL- 6 , IL- 8 , and TNF- $\alpha$ contribute to the progression of sepsis in patients $[6,7]$.
Current sepsis treatment methods consist of treating the underlying infection using broad spectrum antibiotics and remediating hemodynamic changes through fluid resuscitation [8]. Recently, the use of extracorporeal blood purification has been investigated as an alternative approach to sepsis treatment through the removal of substances linked to the pathogenesis of sepsis. Endotoxins have been identified as key substances in sepsis progression, and polymyxin Bimmobilized columns have been designed for the removal of endotoxins through direct hemoperfusion [9]. Despite the successful targeted removal of endotoxins from septic patients, clinical trials have shown an inconclusive benefit in the removal of endotoxins alone $[10,11]$. The removal of inflammatory cytokines has also attracted increasing 


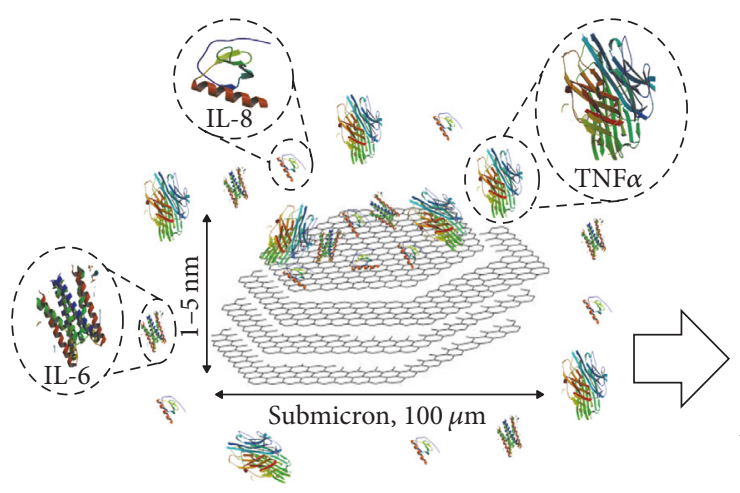

(a)

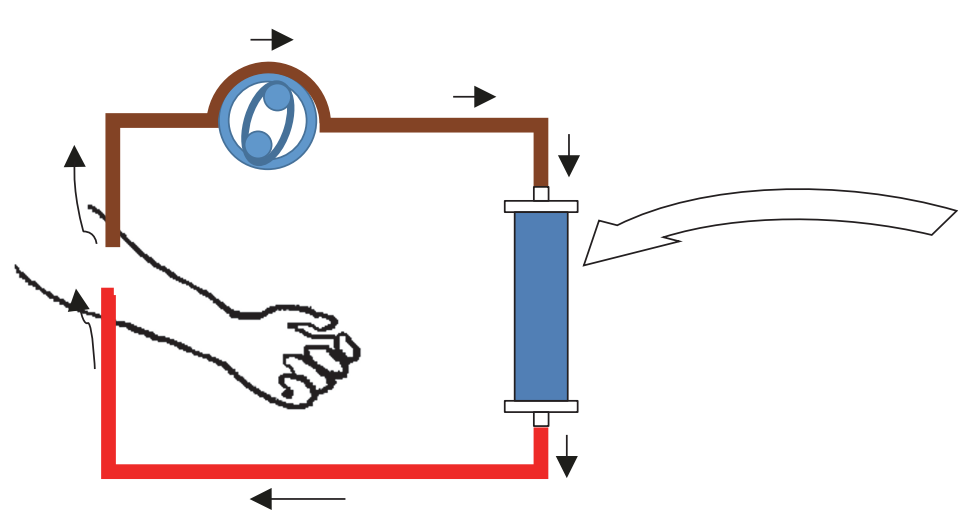

(e)

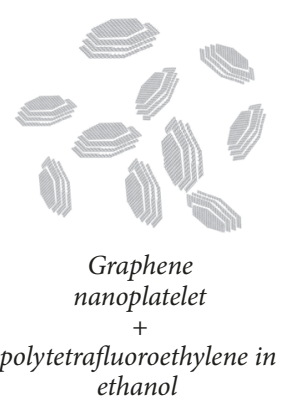

(b)

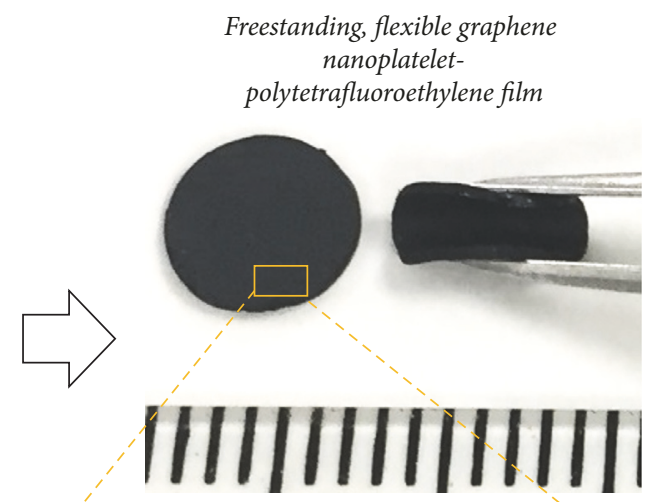

(c)

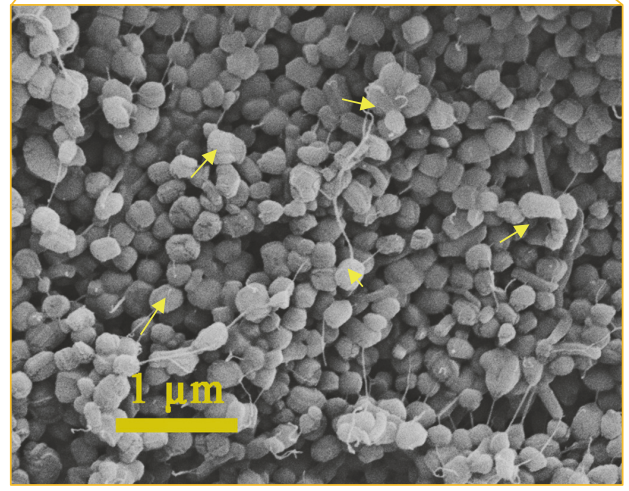

(d)

FIGURE 1: Schematic diagrams showing (a) the layered, open structure of graphene nanoplatelets (GNP) which are suitable for broad spectrum proinflammatory cytokine removal and (b) the use of PTFE, as a binder to produce the (c) freestanding flexible GNP-PTFE film with (d) exposed GNP surfaces and PTFE holding the GNP (indicated by yellow arrow), indicating a potential route for use in (e) hemoperfusion.

attention as an option for sepsis treatment. Polymeric porous adsorbents such as CytoSorb ${ }^{\circledR}$ (CytoSorbents Corporation, USA) have been developed for target removal of molecules between 10 and $50 \mathrm{kDa}$ and have shown some success in both animal studies and clinical case studies [12, 13]. Furthermore, synthetic activated carbon (AC) is also an attractive adsorbent for hemoperfusion systems, as its inert character and tunable porosity (e.g., mesomacroporous texture) allow for optimal adsorption of proteins with different dimensions. Recent work has utilized carbon in the form of AC [14], carbide-derived carbons $[15,16]$, and polymer-pyrolyzed carbon monoliths [17] for use in adsorbing overexpressed cytokines and other toxins inherent in septic patients. These amorphous and graphitic carbons rely on an internally accessible surface area and entrapment of proteins by slitshaped pores for adsorption [18].

While the results of these previous investigations have shown a viable proof-of-concept and efficient adsorption of these cytokines, these adsorbents lack rapid kinetics due to a complex adsorption model $[14,19]$. More advanced synthetic materials have a high manufacturing cost. Graphene nanoplatelets (GNP) are a new, commercially available, lowcost graphene material that consists of stacks of graphene layers $1-5 \mathrm{~nm}$ in thickness and tunable lateral dimensions ranging from submicron scale to over $100 \mu \mathrm{m}$, as determined by milling and processing [20]. GNP have an openly accessible and large specific surface area $\left(\sim 500 \mathrm{~m}^{2} / \mathrm{g}\right)[21]$, making them an ideal material for rapid protein adsorption (Figure 1(a)). Typical GNP synthesis involves mechanical exfoliation of expanded graphite prepared from natural graphite $[22,23]$. Previous report has tested the suitability of expanded graphite materials with inert surface and abundant macropores for large molecule adsorption and bacteriostatic properties and, subsequently, demonstrated potential for medical applications as wound dressings [24]. Owing to the abundant supply and low price of the source natural graphite material, GNP warrants further investigation as a potential cost-effective medical adsorbent. In this paper, we explore the use of noncytotoxic graphene nanoplatelets for rapid removal of a broad spectrum of proinflammatory cytokines identified as instigators of sepsis progression and the preparation of a freestanding flexible GNP-poly(tetrafluoroethylene) film (Figures 1(b) and 1(c)) with preserved surface (Figure 1(d)) and cytokine adsorption characteristics for practical use in hemoperfusion applications (Figure 1(e)).

\section{Materials and Methods}

2.1. Material Synthesis. The GNP used in this study was Grade C-500 xGnPTM obtained from XG Sciences $@$ (Lansing, MI, 
USA). GNP was vacuum annealed $\left(10^{-6}\right.$ torr $)$ at $1800^{\circ} \mathrm{C}$ for eight hours in a vacuum furnace (Solar Atmospheres, PA, USA) to remove functional groups, graphitize the surface of the GNP, and produce vacuum annealed GNP (VA-GNP). VA-GNP-PTFE film was prepared by mixing defunctionalized VA-GNP with PTFE $(60 \% \mathrm{w} / \mathrm{w}$ in water) solution (Sigma-Aldrich, US) in ethanol at a ratio of $19: 1$. Evaporation of ethanol left a homogenous VA-GNP-PTFE dense slurry which was rolled out into a cohesive, freestanding $100 \mu \mathrm{m}$ thick film. The resulting film was then subjected to nitrogen adsorption analysis and cytokine removal assessments to establish its efficacy as a hemoadsorbent.

2.2. Material Characterization. GNP particle size was determined by dynamic light scattering (DLS) using Zetasizer Nano ZS (Malvern, UK). High magnification images of GNP were obtained using transmission electron microscopy (TEM) (JEM2100, JEOL, Japan). The surface and internal porous morphologies of GNP powder and GNP-PTFE film were characterized by scanning electron microscopy (SEM) (Zeiss Supra 50VP field-emission SEM, USA). Quadrasorb pore size analyzer (Quantachrome, FL, USA) was used to measure specific surface area and porosity by carrying out $\mathrm{N}_{2}$ adsorption-desorption measurements at 0.05-0.99 $P / P_{0}$ relative pressures at $77.4 \mathrm{~K}$. The data was analyzed using Quantachrome data analysis software (Quantachrome QuadraWin $^{\mathrm{TM}}$ 5.1). The specific surface areas were calculated using the Brunauer, Emmett, and Teller (BET) method while the pore size distribution was estimated using quenched solid density functional theory (QSDFT) modelling.

2.3. Biocompatibility Assessment. To ensure the safety of GNP, its cytotoxicity was also assessed. The liver, as the main organ for detoxification, has been reported to accumulate nanoparticles after their injection in the bloodstream $[25,26]$. A human hepatic epithelial cell line HepG2 (CRL-11997 ${ }^{\mathrm{TM}}$, ATCC $^{\circledR}$, VA, USA) was used for the assessment of GNP cytotoxicity, with silver nanoparticles as a positive control due to their widely reported cytotoxicity and hepatocytotoxicity $[27,28]$. HepG2 cell viability after treatments was determined by comparing cellular 3-(4,5-dimethylthiazol-2-yl)-5-(3carboxymethoxyphenyl)-2-(4-sulfophenyl)-2H-tetrazolium (MTS) conversion using CellTiter $96^{\circledR}$ Aqueous One Solution Cell Proliferation Assay (Promega, Southampton, UK), while the material cytotoxicity was assessed through the level of lactate dehydrogenase (LDH) released by the HepG2 cells using CytoTox $96^{\circledR}$ Non-Radioactive Cytotoxicity Assay (Promega, Southampton, UK).

2.4. Cytokine Adsorption Experiments. The cytokine marker adsorption profile of GNP was first evaluated by incubating $10 \% \mathrm{v} / \mathrm{v}$ of GNP with fresh frozen human blood plasma (Cambridge Bioscience Ltd., Cambridge, UK) spiked with $1 \mathrm{ng} / \mathrm{mL}$ IL-8, IL-1 $\beta$, IL-6, IL-10, and TNF- $\alpha$ (BD Biosciences, UK) for 60 minutes. Concentrations of the selected cytokine marker were determined using BD Cytometric Bead Array (CBA) Human Inflammatory Cytokines Kit (BD Biosciences, UK). The GNP-PTFE film adsorption kinetics of selected cytokine markers IL-8, IL-6, and TNF- $\alpha$ was compared with PTFE film and GNP powder. The selected cytokine marker concentrations were measured by enzyme-linked immunosorbent assay (ELISA) using BD Biosciences ELISA set. Two-way ANOVA statistical analysis was performed using Prism 6 version 6.05 (GraphPad Software, Inc.).

\section{Results and Discussion}

3.1. Material Morphology and Surface Characteristics. In the untreated GNP samples, loose agglomerates of GNP can be observed under SEM (Figure 2(a)). At a higher magnification, SEM images revealed that these agglomerates consisted of submicron size particles (Figure 2(b)). After sonication in ethanol, smaller agglomerates of GNP and single nanoplatelets were observed under TEM (Figure 2(c)), revealing their multilayer-stacked graphene structure. Under higher magnification, a single nanoplatelet can be observed from a top-down perspective in TEM (Figure 2(c)). VA-GNP showed a 2.7-fold decrease in specific surface area and pore volume as compared to GNP. This was observed along with a 3-fold increase in VA-GNP particle size as compared to GNP determined by dynamic light scattering (DLS) analysis. The decrease in surface area is caused by the restacking of graphene planes during annealing [29]. However, increased agglomeration of defunctionalized hydrophobic VA-GNP in water is mainly responsible for the DLS-observed increase in their particle sizes. SEM and TEM images revealed the small particle size and tightly stacked layered structure of the GNP with high electron density between graphene layers, preventing access of $\mathrm{N}_{2}$ molecules to internal voids in the material. The large BET surface area of the GNP is attributed predominantly to its outer accessible surface area. When VAGNP was bound by PTFE (VA-GNP-PTFE) (Figure 2(d)), the film showed a slight decrease in the adsorbed nitrogen volume (Figure $3(\mathrm{a})$ ) and micropore ( $<2 \mathrm{~nm}$ diameter pores) volume (Figure $3(\mathrm{~b})$ ), while the shape of the isotherm and the overall pore size distribution remained similar to the VA-GNP. The specific surface area and micropore volume of the VA-GNP-PTFE film remained as high as $210 \mathrm{~m}^{2} / \mathrm{g}$ and $0.30 \mathrm{~cm}^{3} / \mathrm{g}$, respectively, and only slightly lower than the VAGNP alone at $294 \mathrm{~m}^{2} / \mathrm{g}$ and $0.39 \mathrm{~cm}^{3} / \mathrm{g}$, respectively (Table 1 ). This decrease in the specific surface area and micropore volume measured by the nitrogen adsorption analysis could be attributed to the addition of nonporous PTFE polymer that reduced the accessible VA-GNP surface to $\mathrm{N}_{2}$ molecules.

3.2. Cytokine Markers Removal Profile of GNP. Following materials characterization, cytokine adsorption efficacy of the GNP-PTFE film was subsequently assessed. The cytokine marker adsorption profile of GNP showed rapid and efficient removal of cytokines from human plasma spiked with a cytokine cocktail. The concentration of smaller cytokines IL-8 $(8 \mathrm{kDa})$ and IL-1 $\beta(17 \mathrm{kDa})$ in the spiked plasma was reduced from over $1500 \mathrm{pg} / \mathrm{mL}$ to $20 \mathrm{pg} / \mathrm{mL}$ within $5 \mathrm{~min}$ utes of direct contact (Figure 4(a)). In comparison, GNP removal of larger cytokine markers IL-10 $(18.5 \mathrm{kDa})$ and IL-6 $(20.5 \mathrm{kDa})$ by GNP appeared to be slightly slower. However, 


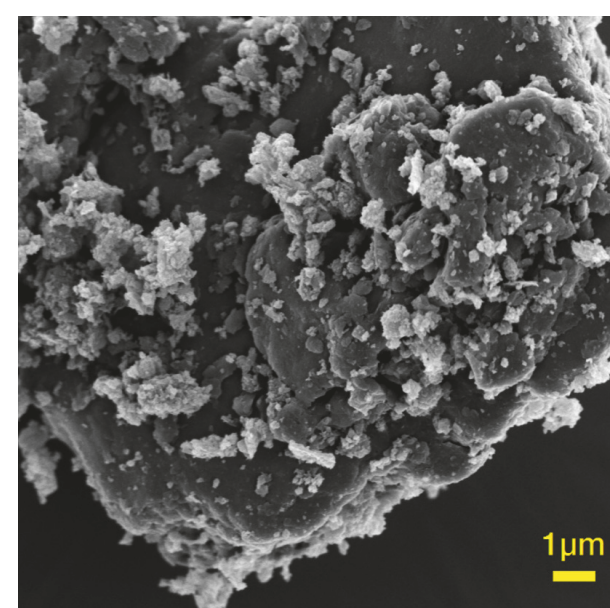

(a)

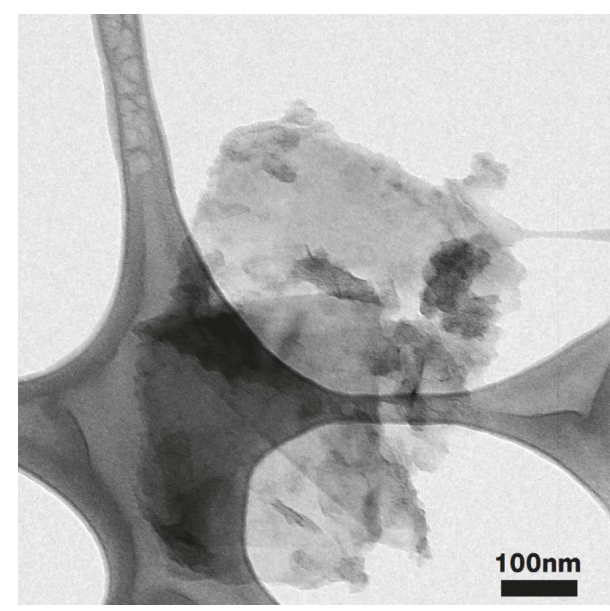

(c)

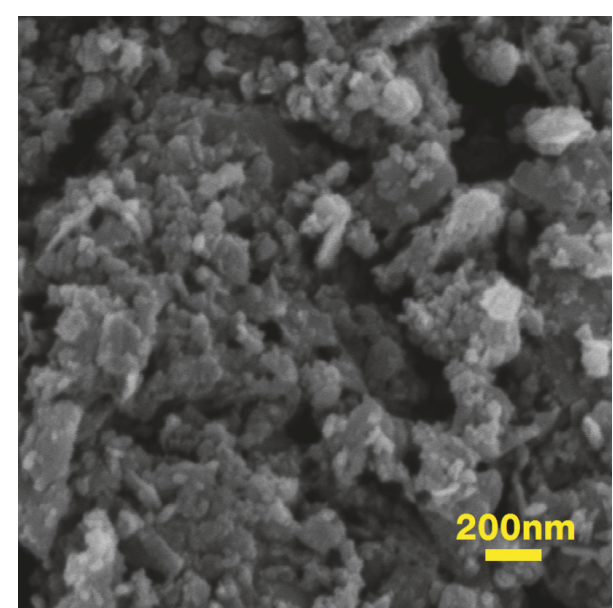

(b)

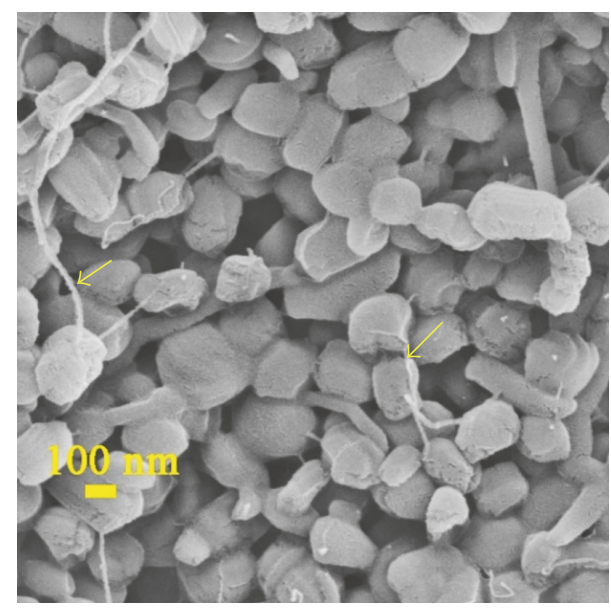

(d)

FIGURE 2: (a) SEM image of a GNP agglomerate; (b) higher magnification SEM image revealing submicron GNP particles; (c) TEM image of a single GNP, indicating a single GNP particle consists of stacks of graphene layers; and (d) SEM image of the GNP-PTFE film in which GNP particles were held together by PTFE (indicated by arrows).

TABLE 1: Porosity of GNP, VA-GNP, and VA-GNP-PTFE film determined using low temperature nitrogen adsorption analysis. Sample specific surface area $\left(S_{\mathrm{BET}}\right)$ and pore volume $\left(V_{\mathrm{QSDFT}}\right)$ /size $\left(D_{\text {mode }}\right)$ were calculated using BET and QSDFT, respectively. Equivalent particle diameter (d) was determined using dynamic light scattering.

\begin{tabular}{|c|c|c|c|c|}
\hline Sample & $S_{\mathrm{BET}}\left(\mathrm{m}^{2} / \mathrm{g}\right)$ & $V_{\mathrm{QSDFT}}\left(\mathrm{cm}^{3} / \mathrm{g}\right)$ & $D_{\text {mode }}(\mathrm{nm})$ & $d(\mathrm{~nm})$ \\
\hline Initial GNP & 797 & 0.807 & 0.785 & 547 \\
\hline VA-GNP & 294 & 0.397 & 0.723 & 1670 \\
\hline VA-GNP-PTFE & 210 & 0.301 & 0.852 & -- \\
\hline
\end{tabular}

even in these cases $60 \%$ and $50 \%$ removal, respectively, were achieved within the first $5 \mathrm{~min}$ of contact. The TNF- $\alpha$ trimer $(51 \mathrm{kDa})$ is, reportedly, the most challenging molecule to remove in conventional blood purification techniques using carbon sorbents [30-32]. However, a reduction in the plasma TNF- $\alpha$ concentration from $868 \mathrm{pg} / \mathrm{mL}$ to $55 \mathrm{pg} / \mathrm{mL}$ after 5 min contact with GNP indicated a rapid and efficient removal of the TNF- $\alpha$ by GNP. The fast adsorption kinetics can be attributed to direct contact with a completely accessible surface area and minimal diffusion barriers to and on the surface. These results demonstrated GNP's potential for broad spectrum cytokine removal.

3.3. Biocompatibility of GNP. The LDH and MTT cytotoxicity assay results indicated that at lower tested concentrations GNP caused no or minimal cytotoxic effect $(<20 \%$ cytotoxicity and $>80 \%$ cell viability) towards HepG 2 cells, while the silver nanoparticles were cytotoxic at concentrations as low as $0.01 \%(\mathrm{v} / \mathrm{v})$ (Figures $4(\mathrm{~b})$ and $4(\mathrm{c})$ ). HepG2 cell viability fell below $60 \%$ after $0.06 \%$ GNP treatment, indicating a 


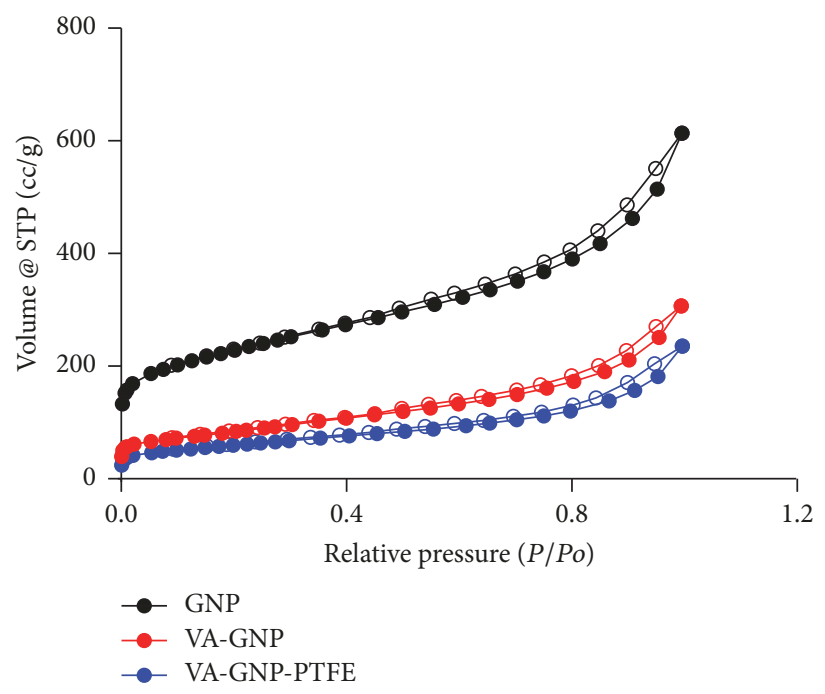

(a)

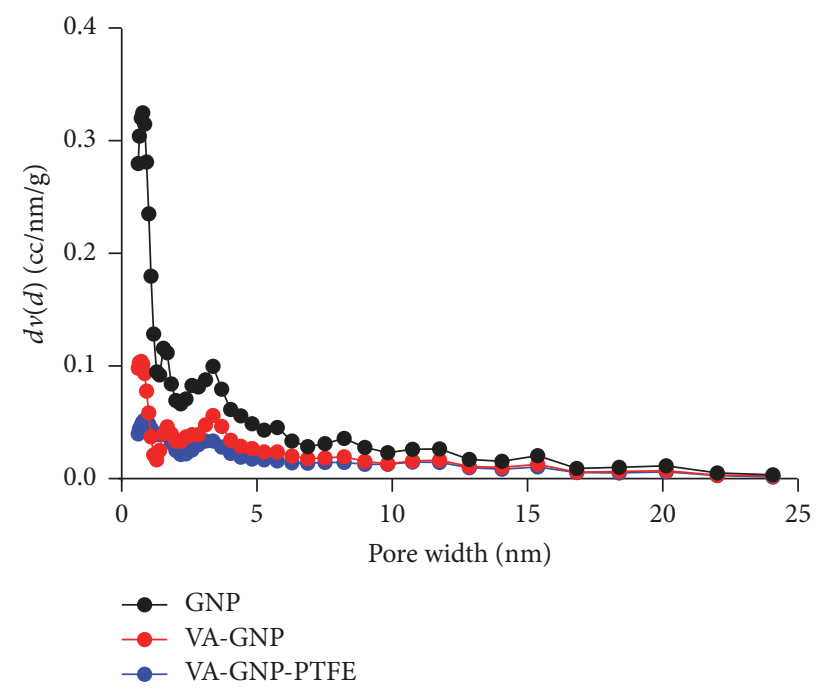

(b)

FIGURE 3: (a) Low temperature nitrogen adsorption isotherms (closed circles denote the adsorption branch and open circles denote the desorption branch) and (b) pore size distributions of the GNP, VA-GNP, and VA-GNP-PTFE film.

suppression of metabolic activity (Figure 4(b)). The cytotoxicity of GNP determined using LDH assay appeared to be dose-dependent and was significantly $(p<0.01)$ lower than the silver nanoparticles at the same concentration (Figure 4(c)). The slight cytotoxic effects of GNP at the concentrations of $0.03 \%$ and $0.06 \%$ are likely attributed to the large absolute volume of GNP in the two-dimensional culture system, which blocked cellular access to vital oxygen and nutrients.

3.4. Cytokine Markers Removal Efficiency of GNP-PTFE Film. The adsorption data for cytokine markers IL- 8 and IL- 6 and TNF- $\alpha$ revealed that the PTFE film alone did not reduce the cytokine concentration in the spiked plasma compared to the control within the 90 min incubation cycle. In contrast, when the spiked plasma was incubated with the GNP particles, IL8 concentration reduced from $633 \mathrm{pg} / \mathrm{mL}$ to $7 \mathrm{pg} / \mathrm{mL}$ within $5 \mathrm{~min}$. IL- 6 decreased from $477 \mathrm{pg} / \mathrm{mL}$ to $22 \mathrm{pg} / \mathrm{mL}$ after $5 \mathrm{~min}$ and further dropped to $8 \mathrm{pg} / \mathrm{mL}$ after $30 \mathrm{~min}$ (Figures 5(a) and 5(b)). The VA-GNP-PTFE film showed a slower and slightly less efficient adsorption of IL-8 from the spiked plasma as compared to the GNP, but with 95\% removal of IL-8 over 90 min of incubation (Figure 5(a)). A significantly lower adsorption efficacy of IL- 6 was observed with VA-GNP-PTFE film as compared to the GNP particles, but VA-GNP-PTFE film removed over $50 \%$ of IL- 6 from the spiked plasma over the $90 \mathrm{~min}$ incubation period (Figure 5(b)). Incorporation of GNP into the PTFE film reduced TNF- $\alpha$ removal compared to the GNP particles alone (Figure 5(c)). This could be due to the packing of GNP particles, which reduced the exposed particle outer surface area. Alternatively, PTFE limited accessibility between the particles and potentially contributed to this effect. We have previously highlighted the need for pores with diameters that exceed protein molecule size [33]. From a practical perspective, this translated to meso-/macropores in activated carbon adsorbents with predominant $70-120 \mathrm{~nm}$ diameter pores as necessary for significant TNF- $\alpha$ adsorption $[17,34,35]$. Although GNP with a similar specific surface area did not feature such large pores, it still demonstrated superior TNF- $\alpha$ adsorption, which was reduced after incorporation of PTFE. This indicated the benefit of GNP's large, outer surface area and underscored the importance of accessible surface just as much as the internal porosity of ACs. Overall, the incorporation of GNP in the PTFE film largely preserved the GNP surface for the adsorption of mid-range molecular weight cytokine markers IL- 6 and IL- 8 and demonstrated significantly lower removal capacity of plasma TNF- $\alpha$ as compared to unbound GNP. Future experiments may further improve GNP surface area exposure by preparing VA-GNPPTFE film with reduced thickness and thus improve TNF$\alpha$ adsorption. This development could yield significant cost benefits to optimize and use GNP for sepsis in blood detoxification devices.

Novel materials developed for direct blood contacting applications often raise hemocompatibility concerns such as fine particle release and blood cell activation. Several of our previously published studies have demonstrated the safe use of carbon-based materials for direct blood contacting applications [34, 36, 37]. Our efforts have developed a commercially viable composite material that utilized PTFE not only to produce a flexible freestanding film structure, but also to hold the GNP particles in place, eliminating fine particle release. In addition to the noncytotoxic nature of the GNP established in this study, further research that delves into the assessments of material hemocompatibility will be beneficial. In particular, future efforts must ensure that the use of this cost-effective adsorbent does not trigger complement cascade, excessive platelet adhesion/activation, or granulocyte activation in ex vivo setups. The GNP-PTFE adsorbent could also be used in the plasma circuit of systems 


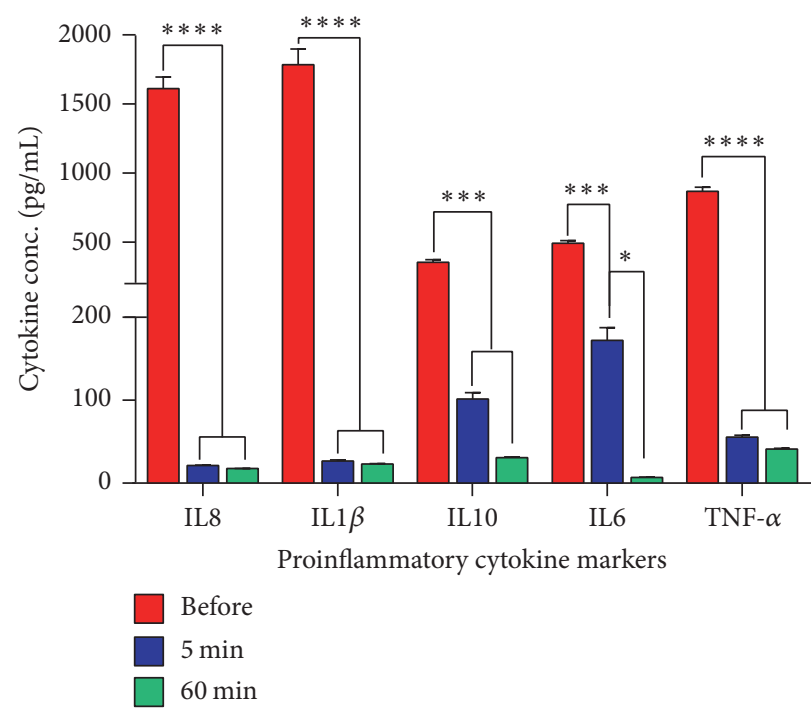

(a)

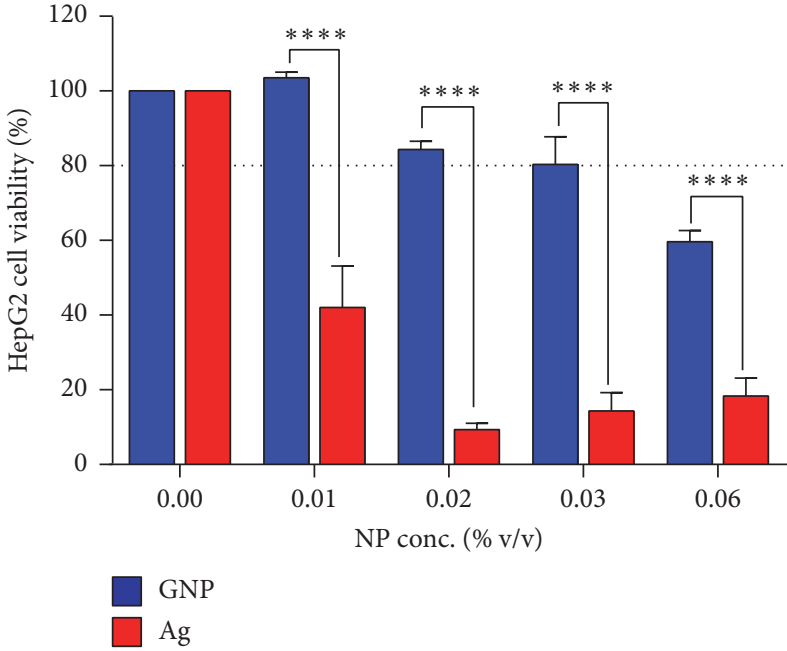

(b)

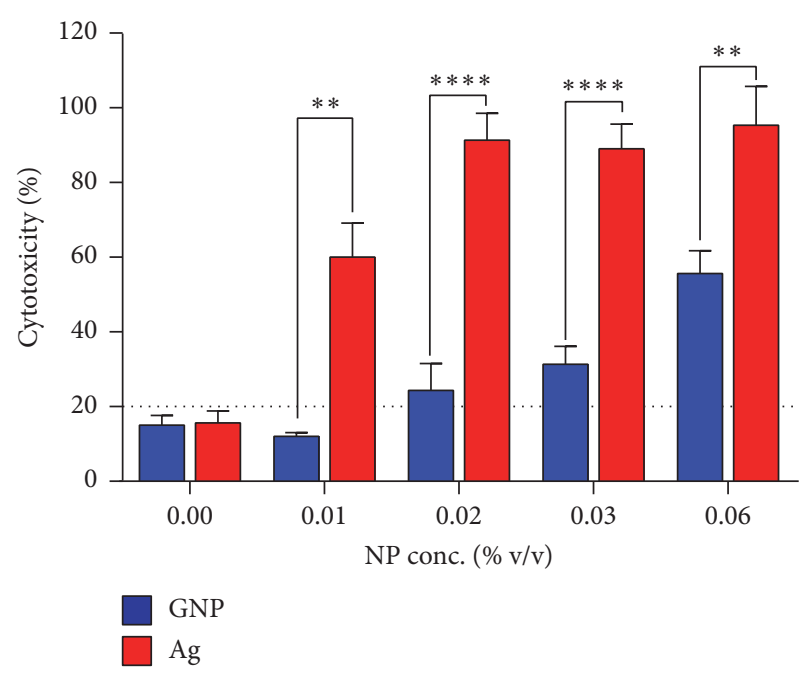

(c)

FIGURE 4: (a) The removal efficiency of inflammatory cytokine markers in spiked human plasma samples; GNP cytotoxicity assessments after 24 hours of direct contact using (b) MTS and (c) LDH assays. * represents $p<0.05$; $* *$ represents $p<0.01$; $* * *$ represents $p<0.001$; $* * * *$ represents $p<0.0001$ estimated using two-way ANOVA statistic test ( \pm standard error of mean, $n=3$ ).

such as MARS ${ }^{\circledR}[38]$ or Prometheus ${ }^{\circledR}[39]$ where adsorbent materials do not come into direct contact with patient blood and, therefore, mitigate issues of blood cell activation and hemocompatibility.

\section{Conclusions}

In summary, we have demonstrated the use of GNP as a fast and very efficient adsorbent with low cytotoxicity for rapid removal of proinflammatory cytokines including IL8 , IL-1 $\beta$, IL-6, IL-10, and TNF- $\alpha$ from human plasma on a clinically relevant scale. The material reported in this study shows faster adsorption compared to all other carbons reported so far, combined with a very high sorption capacity.
Furthermore, we also developed a flexible freestanding GNPPTFE composite material with high accessible surface area for targeted adsorption of cytokines and showed its potential as hemoperfusion adsorbent. Further development of these graphene-based sorbents should aim to confirm their ex vivo hemocompatibility. Subsequently, future research efforts will enhance accessible surface area for improved adsorption of the high molecular weight cytokines including TNF$\alpha$ and bring forth a cost-effective materials solution for hemoperfusion systems for treatment of sepsis.

\section{Conflicts of Interest}

The authors declare that there are no conflicts of interest regarding the publication of this paper. 


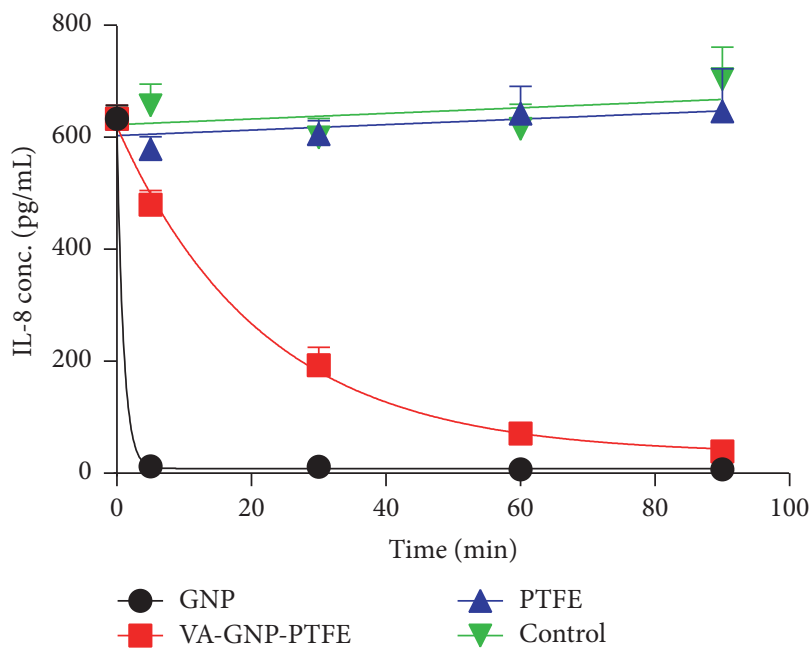

(a)

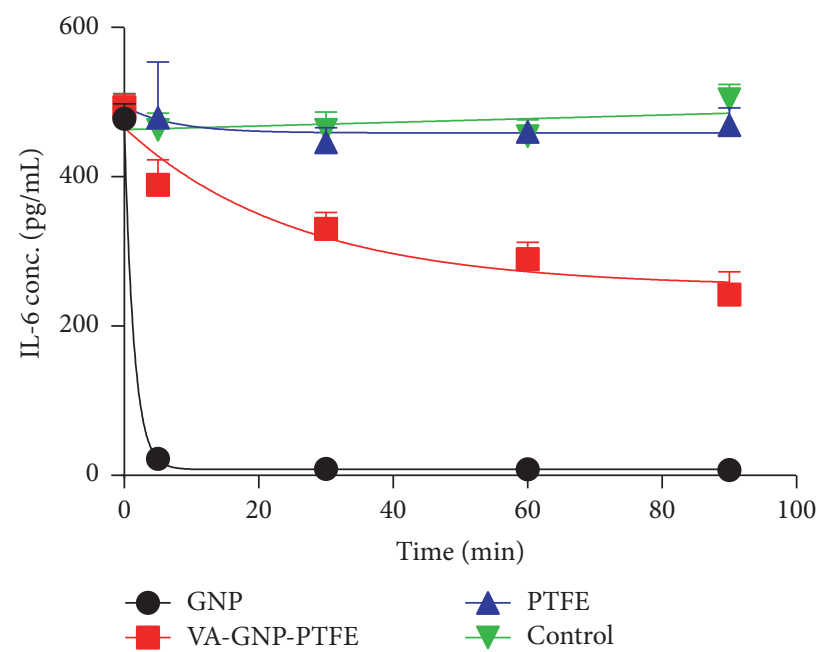

(b)

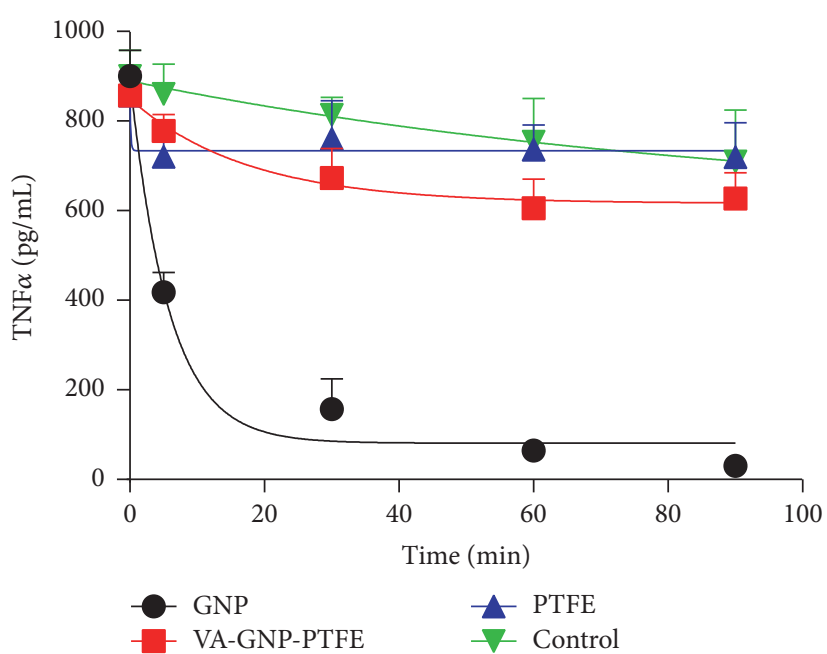

(c)

FIGURE 5: Removal efficiency of inflammatory cytokine markers IL-8 (a), IL-6 (b), and TNF- $\alpha$ (c) from spiked human plasma sample of GNP, VA-GNP-PTFE film, and plain PTFE film $( \pm$ SEM, $n=3)$.

\section{Acknowledgments}

This material is based upon work carried out by the NOMAD project supported by the British Council and the UK Department for Business, Innovation and Skills through the Global Innovation Initiative.

\section{References}

[1] V. Y. Dombrovskiy, A. A. Martin, J. Sunderram, and H. L. Paz, "Rapid increase in hospitalization and mortality rates for severe sepsis in the United States: a trend analysis from 1993 to 2003," Critical Care Medicine, vol. 35, no. 5, pp. 1244-1250, 2007.

[2] A. R. Bedford Russell, "Neonatal sepsis," Paediatrics and Child Health (United Kingdom), vol. 25, no. 6, pp. 271-275, 2015.

[3] B. Tiru, E. K. DiNino, A. Orenstein et al., "The economic and humanistic burden of severe sepsis," PharmacoEconomics, vol. 33, no. 9, pp. 925-937, 2015.
[4] N. K. J. Adhikari, R. A. Fowler, S. Bhagwanjee, and G. D. Rubenfeld, "Critical care and the global burden of critical illness in adults," The Lancet, vol. 376, no. 9749, pp. 1339-1346, 2010.

[5] D. C. Angus and T. van der Poll, "Severe sepsis and septic shock," The New England Journal of Medicine, vol. 369, no. 9, pp. 840851, 2013.

[6] K. Baghel, R. N. Srivastava, A. Chandra et al., "TNF- $\alpha$, IL6 , and IL- 8 cytokines and their association with TNF- $\alpha$ 308 G/A polymorphism and postoperative sepsis," Journal of Gastrointestinal Surgery, vol. 18, no. 8, pp. 1486-1494, 2014.

[7] B. G. Chousterman, F. K. Swirski, and G. F. Weber, "Cytokine storm and sepsis disease pathogenesis," Seminars in Immunopathology, vol. 39, no. 5, pp. 517-528, 2017.

[8] A. M. Taeb, M. H. Hooper, and P. E. Marik, "Sepsis: Current definition, pathophysiology, diagnosis, and management," Nutrition in Clinical Practice, vol. 32, no. 3, pp. 296-308, 2017.

[9] H. Aoki, M. Kodama, T. Tani, and K. Hanasawa, "Treatment of sepsis by extracorporeal elimination of endotoxin using 
polymyxin B-inimobilized fiber," The American Journal of Surgery, vol. 167, no. 4, pp. 412-417, 1994.

[10] C. Ronco and D. J. Klein, "Polymyxin B hemoperfusion: A mechanistic perspective," Critical Care, vol. 18, no. 3, article no. 309, 2014.

[11] T. Terayama, K. Yamakawa, Y. Umemura, M. Aihara, and S. Fujimi, "Polymyxin B hemoperfusion for sepsis and septic shock: A systematic review and meta-analysis," Surgical Infections, vol. 18, no. 3, pp. 225-233, 2017.

[12] T. Taniguchi, F. Hirai, Y. Takemoto et al., "A novel adsorbent of circulating bacterial toxins and cytokines: The effect of direct hemoperfusion with CTR column for the treatment of experimental endotoxemia," Critical Care Medicine, vol. 34, no. 3, pp. 800-806, 2006.

[13] K. Kogelmann, D. Jarczak, M. Scheller, and M. Drüner, "Hemoadsorption by CytoSorb in septic patients: a case series," Critical Care, vol. 21, no. 1, 2017.

[14] S. Inoue, K. Kiriyama, Y. Hatanaka, and H. Kanoh, "Adsorption properties of an activated carbon for 18 cytokines and HMGB1 from inflammatory model plasma," Colloids and Surfaces B: Biointerfaces, vol. 126, pp. 58-62, 2015.

[15] S. Yachamaneni, G. Yushin, S.-H. Yeon et al., "Mesoporous carbide-derived carbon for cytokine removal from blood plasma," Biomaterials, vol. 31, no. 18, pp. 4789-4794, 2010.

[16] V. Presser, S. Yeon, C. Vakifahmetoglu et al., "Hierarchical porous carbide-derived carbons for the removal of cytokines from blood plasma," Advanced Healthcare Materials, vol. 1, no. 6, pp. 682-682, 2012.

[17] S. R. Sandeman, C. A. Howell, S. V. Mikhalovsky et al., "Inflammatory cytokine removal by an activated carbon device in a flowing system," Biomaterials, vol. 29, no. 11, pp. 1638-1644, 2008.

[18] S. V. Mikhalovsky, "Emerging technologies in extracorporeal treatment: Focus on adsorption," Perfusion, vol. 18, no. 1, pp. 4754, 2003.

[19] V. M. Gun'Ko, V. V. Turov, O. P. Kozynchenko et al., "Activation and structural and adsorption features of activated carbons with highly developed micro-, meso- and macroporosity," Adsorption, vol. 17, no. 3, pp. 453-460, 2011.

[20] X. Li, S. Biswas, and L. T. Drzal, "High temperature vacuum annealing and hydrogenation modification of exfoliated graphite nanoplatelets," Journal of Engineering (United States), vol. 2013, Article ID 638576, 2013.

[21] L. T. Drzal, Graphene Nanoplatelets: A Muli-Functional Nanomaterial Additive for Polymers and Composites, I. XG Sciences, 2015.

[22] S. Y. Choi, M. Mamak, E. Cordola, and U. Stadler, "Large scale production of high aspect ratio graphite nanoplatelets with tunable oxygen functionality," Journal of Materials Chemistry, vol. 21, no. 13, pp. 5142-5147, 2011.

[23] A. V. Melezhyk and A. G. Tkachev, "Synthesis of graphene nanoplatelets from peroxosulfate graphite intercalation compounds," Nanosystems: Physics, Chemistry, Mathematics, vol. 5, no. 2, p. 13, 2014.

[24] W. Shen, S. Wen, N. Cao et al., "Expanded graphite - a new kind of biomedical material," Carbon, vol. 37, no. 2, pp. 356-358, 1999.

[25] S. Hirn, M. Semmler-Behnke, C. Schleh et al., "Particle sizedependent and surface charge-dependent biodistribution of gold nanoparticles after intravenous administration," European Journal of Pharmaceutics and Biopharmaceutics, vol. 77, no. 3, pp. 407-416, 2011.
[26] N. Kurantowicz, B. Strojny, E. Sawosz et al., "Biodistribution of a high dose of diamond, graphite, and graphene oxide nanoparticles after multiple intraperitoneal injections in rats," Nanoscale Research Letters, vol. 10, no. 1, article no. 398, 2015.

[27] Y. Xue, T. Zhang, B. Zhang, F. Gong, Y. Huang, and M. Tang, "Cytotoxicity and apoptosis induced by silver nanoparticles in human liver HepG2 cells in different dispersion media," Journal of Applied Toxicology, vol. 36, no. 3, pp. 352-360, 2016.

[28] B. K. Gaiser, S. Hirn, A. Kermanizadeh et al., "Effects of silver nanoparticles on the liver and hepatocytes in vitro," Toxicological Sciences, vol. 131, no. 2, pp. 537-547, 2013.

[29] S. Osswald, J. Chmiola, and Y. Gogotsi, "Structural evolution of carbide-derived carbons upon vacuum annealing," Carbon, vol. 50, no. 13, pp. 4880-4886, 2012.

[30] B. R. Glick, T. L. Delovitch, and C. L. Patten, "9.1.3 tumor necrosis factor," in Medical Biotechnology, American Society for Microbiology (ASM).

[31] P. Heering, S. Morgera, F. J. Schmitz et al., "Cytokine removal and cardiovascular hemodynamics in septic patients with continuous venovenous hemofiltration," Intensive Care Medicine, vol. 23, no. 3, pp. 288-296, 1997.

[32] S. Harm, F. Gabor, and J. Hartmann, "Characterization of adsorbents for cytokine removal from blood in an in vitro model," Journal of Immunology Research, vol. 2015, Article ID 484736, 2015.

[33] G. Yushin, E. N. Hoffman, M. W. Barsoum et al., "Mesoporous carbide-derived carbon with porosity tuned for efficient adsorption of cytokines," Biomaterials, vol. 27, no. 34, pp. 5755-5762, 2006.

[34] C. A. Howell, S. R. Sandeman, G. J. Phillips et al., "Nanoporous activated carbon beads and monolithic columns as effective hemoadsorbents for inflammatory cytokines," The International Journal of Artificial Organs, vol. 36, no. 9, pp. 624-632, 2013.

[35] S. R. Tennison et al., "Carbon and its use in blood cleansing applications,” US Pat., 20130072845 A1, 2013.

[36] S. R. Sandeman, Y. Zheng, G. C. Ingavle et al., "A haemocompatible and scalable nanoporous adsorbent monolith synthesised using a novel lignin binder route to augment the adsorption of poorly removed uraemic toxins in haemodialysis," Biomedical Materials, vol. 12, no. 3, Article ID 035001, 2017.

[37] S. R. Sandeman, C. A. Howell, G. J. Phillips et al., "An adsorbent monolith device to augment the removal of uraemic toxins during haemodialysis," Journal of Materials Science: Materials in Medicine, vol. 25, no. 6, pp. 1589-1597, 2014.

[38] S. R. Mitzner, J. Stange, S. Klammt, S. Koball, H. Hickstein, and E. C. Reisinger, "Albumin dialysis MARS: Knowledge from 10 years of clinical investigation," ASAIO Journal, vol. 55, no. 5, pp. 498-502, 2009.

[39] M. Oppert, S. Rademacher, K. Petrasch, and A. Jörres, "Extracorporeal liver support therapy with prometheus in patients with liver failure in the intensive care unit," Therapeutic Apheresis and Dialysis, vol. 13, no. 5, pp. 426-430, 2009. 


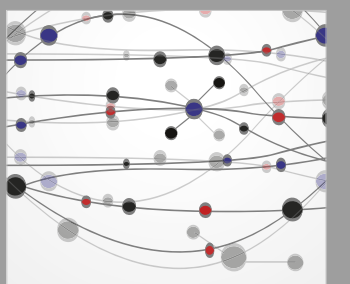

The Scientific World Journal
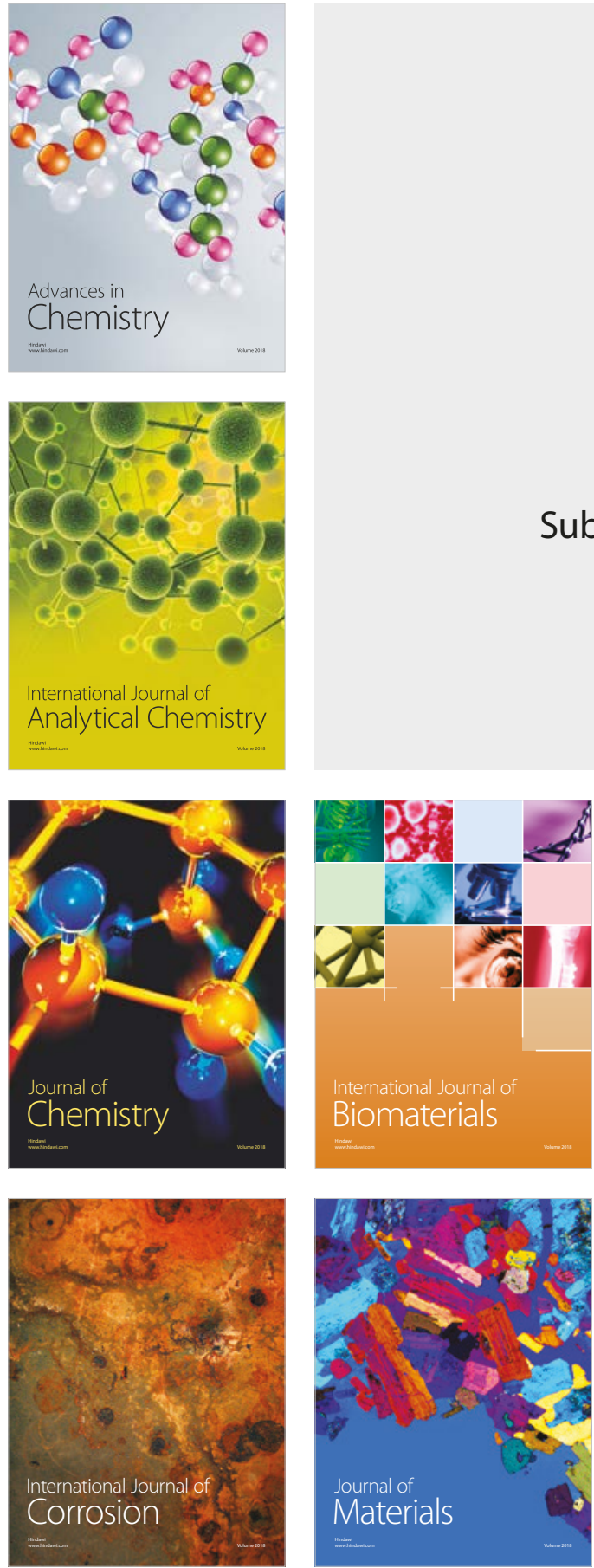

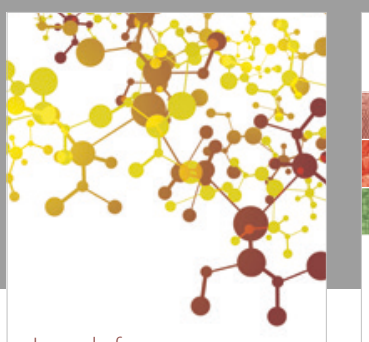

Journal of

Applied Chemistry
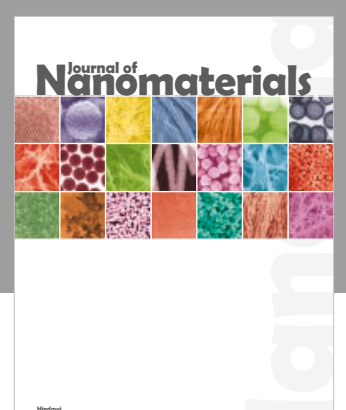

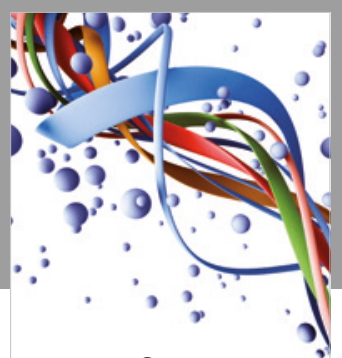

Scientifica

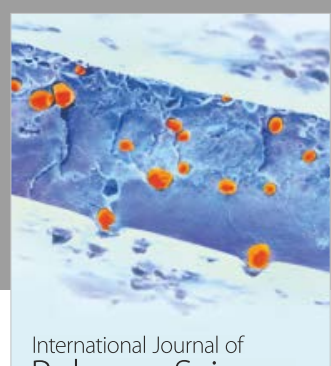

Polymer Science

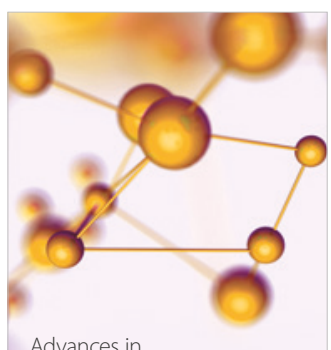

Physical Chemistry
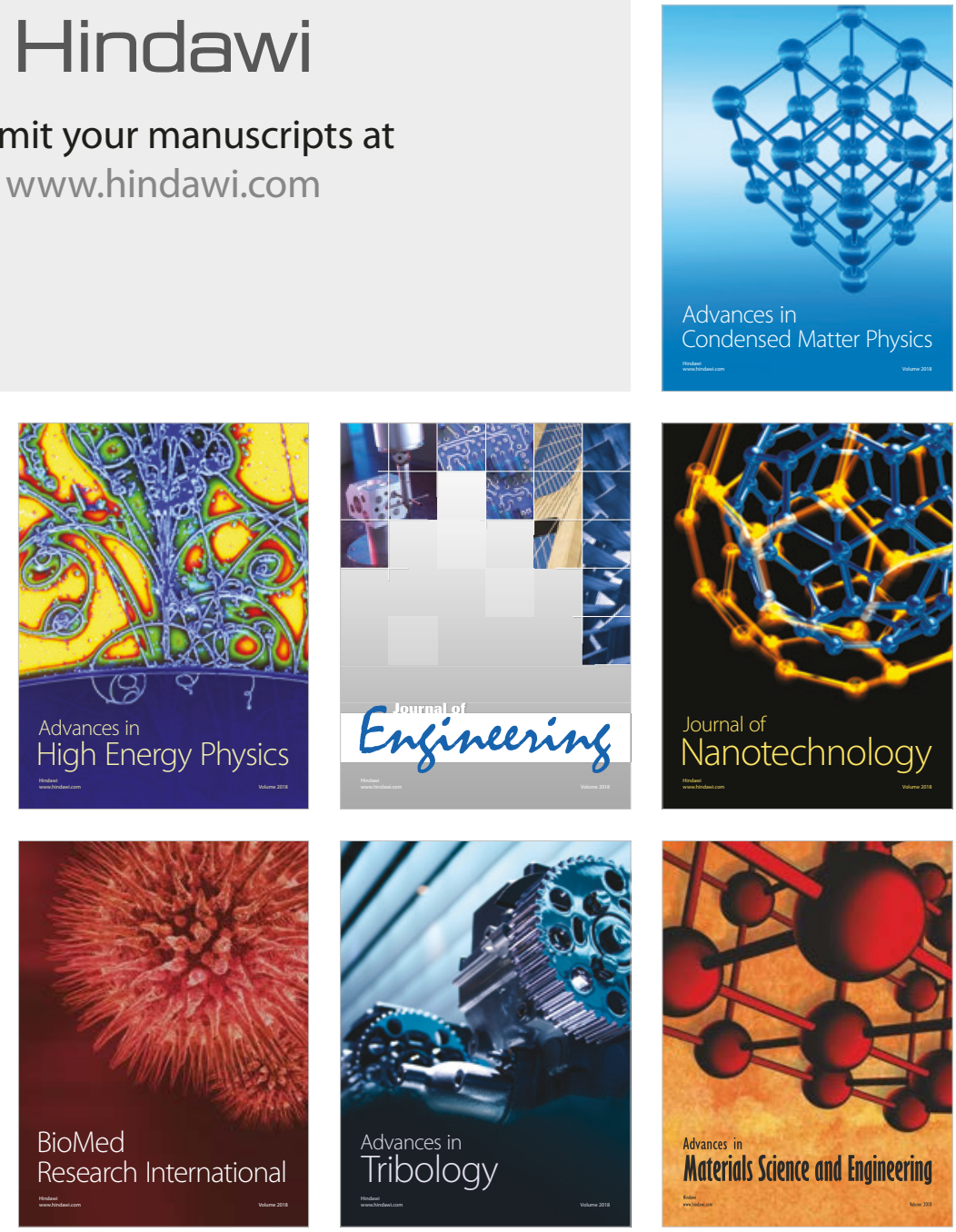